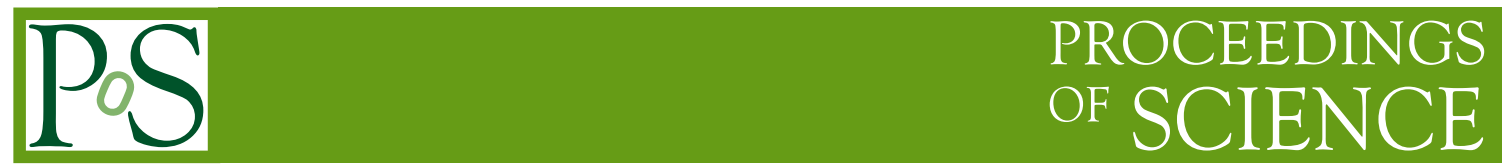

\title{
Latest results from the LHC
}

\author{
Lucia Masetti* \\ PRISMA Cluster of Excellence and Institute for Physics \\ Johannes Gutenberg University Mainz \\ E-mail: lucia.masetti@cern.ch
}

After its first 3 years of operation with proton-proton collisions at a centre-of-mass energy of 7 or $8 \mathrm{TeV}$, the Large Hadron Collider (LHC) at CERN and its experiments are being upgraded to restart in 2015 with a centre-of-mass energy of 13-14 TeV. The first observation of the Higgs boson in 2012 was surely the most popular achievement of the LHC, but the list of interesting results is much longer. A very personal selection of recent results is presented here.

31st International Symposium on Lattice Field Theory - LATTICE 2013

July 29 - August 3, 2013

Mainz, Germany

${ }^{*}$ Speaker. 


\section{Introduction}

In the first 3 years of running both the LHC and its experiments achieved performances beyond expectation, allowing the particle physics community to produce a wealth of high quality results in a very short time.

\subsection{Accelerator}

In 2010 and 2011 the LHC collided two proton beams, each consisting of 1380 bunches of protons spaced by $50 \mathrm{~ns}$, at a centre-of-mass energy of $7 \mathrm{TeV}$. Each bunch consisted of up to $1.45 \times 10^{11}$ protons, leading to a peak luminosity of $3.7 \times 10^{33} \mathrm{~cm}^{-2} \mathrm{~s}^{-1}$. In 2012 the centre-ofmass energy was raised to $8 \mathrm{TeV}$ and the improved focussing allowed for a higher bunch intensity and a peak luminosity of $7.7 \times 10^{33} \mathrm{~cm}^{-2} \mathrm{~s}^{-1}$ with the same bunch spacing. After the shutdown, the LHC will restart operation in 2015 with the aim to reach the nominal centre-of-mass energy of $14 \mathrm{TeV}$ and a peak luminosity of $10^{34} \mathrm{~cm}^{-2} \mathrm{~s}^{-1}$ with less intense bunches at $25 \mathrm{~ns}$ spacing.

\subsection{Experiments}

The results presented in the following sections were obtained by the experiments ATLAS, CMS and LHCb: 3 of the 4 main experiments at the LHC. ATLAS and CMS are general purpose detectors with $4 \pi$ coverage and cylindric geometry. Although most detector components are based on different technologies, they have similar structure and performance: a coverage up to a pseudorapidity $(\eta)$ of about 5 in both beam directions, vertex resolution of $15 \mu \mathrm{m}$, energy resolution of $O(1 \%)$ for electrons and of $O(10 \%)$ for pions with a transverse energy of $100 \mathrm{GeV}$ and momentum resolution of less than 5-10\% for muons with a transverse momentum up to $1 \mathrm{TeV}$. The LHCb detector was build specifically for the needs of $b$ physics and it covers therefore only the forward region between 2 and 5 in $\eta$. Its vertex resolution is better than the one of ATLAS and CMS and additional components provide particle identification for electrons, pions, kaons and protons, while ATLAS can only distinguish between electrons and pions and CMS has no specific detectors for particle identification.

\subsection{Challenges and results}

In spite of the lower centre-of-mass energy and double bunch spacing with respect to the design, the LHC could almost reach the design peak luminosity by increasing the bunch intensity and by improving the focussing. This lead to an increase in the number of simultaneous protonproton collisions (pile-up) in each event, which in 2012 went up to 40 with an average value of 21. At the LHCb interaction point the peak luminosity was reduced to keep the pile-up to a lower level. Coping with pile-up has been the main challenge for the experiments, but their excellent vertex resolution allowed for a good separation between the different collisions.

Between March and July 2013 ATLAS, CMS and LHCb published a total of 76 refereed articles and 125 new preliminary results were presented at conferences, many of which include also the high pile-up data collected in 2012. 

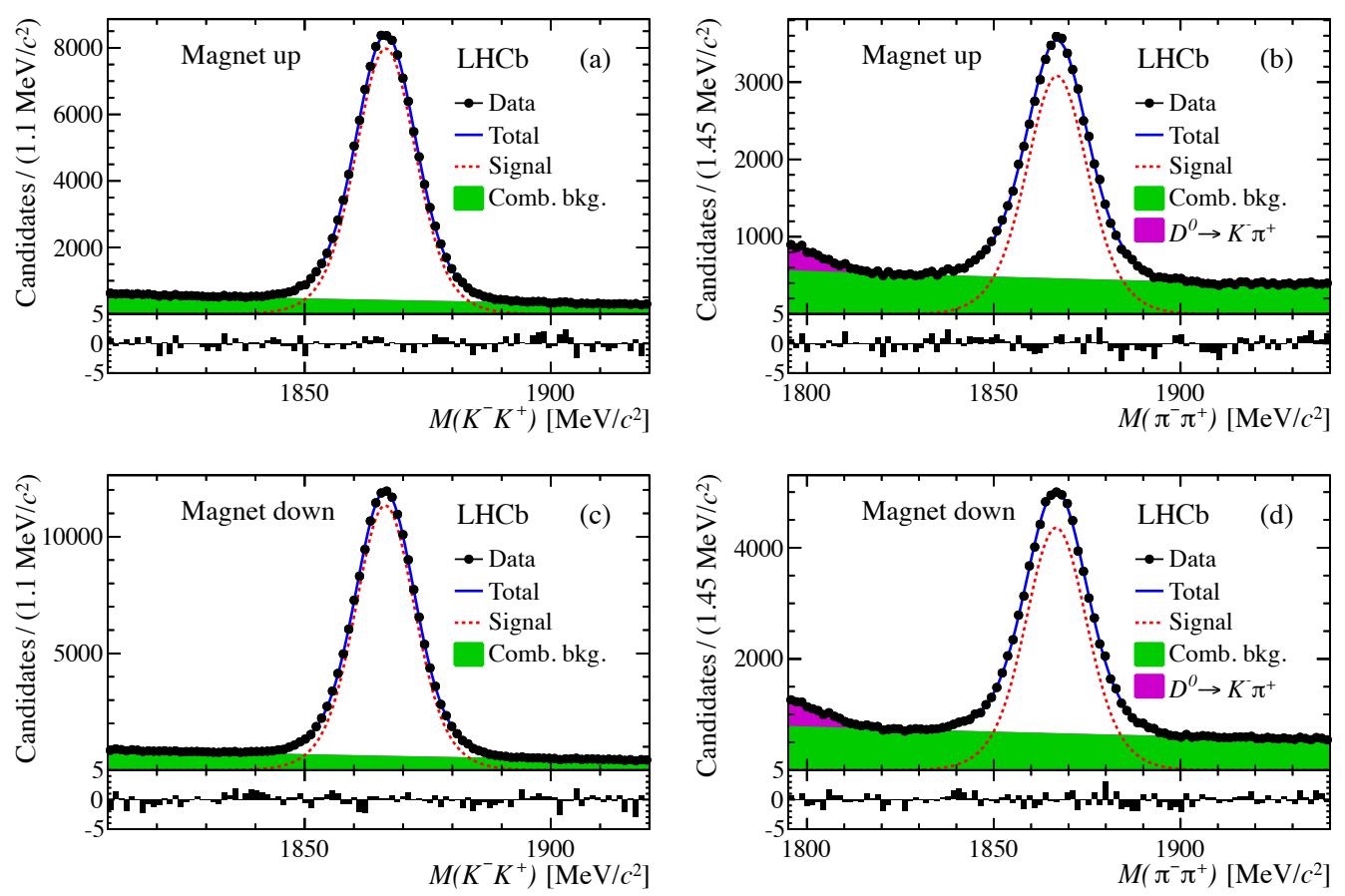

Figure 1: Invariant mass distributions for $D^{0} \rightarrow K^{-} K^{+}$(a, c) and $D^{0} \rightarrow \pi^{-} \pi^{+}$(b, d) muon-tagged candidates for the two magnet polarities. Underneath each plot the pull in each mass bin is shown [1].

\section{Flavour physics}

Recently many precision measurements in the field of flavour physics were published not only by LHCb, but also by ATLAS and CMS. Old tensions with the theoretical predictions were solved, new ones appeared and in general a better knowledge of the $B_{s}$ mesons was obtained.

\subsection{Search for direct $C P$ violation in charm mesons}

A search for direct $C P$ violation in $D^{0} \rightarrow K^{+} K^{-}$and $D^{0} \rightarrow \pi^{+} \pi^{-}$decays was performed by $\mathrm{LHCb}$ using data corresponding to an integrated luminosity of $1.0 \mathrm{fb}^{-1}$ collected in 2011 at a centre-of-mass energy of $7 \mathrm{TeV}$. $D^{0}$ mesons produced in inclusive semileptonic $b$-hadron decays to the $D^{0} \mu X$ final state were selected and the charge of the accompanying muon was used to tag their flavour. Muon detection and effective production asymmetries between $D^{0}$ and $\bar{D}^{0}$ cancel in the difference between the $K K$ and $\pi \pi$ asymmetries, reducing the systematic uncertainty on the result. Furthermore data with different magnet polarity were analysed separately (see Fig. 1) and averaged. The difference in the $C P$-violating asymmetries between the two decay channels, which corresponds to the difference in direct $C P$-violation asymmetries with negligible corrections, was measured to be [1].

$$
\Delta A_{C P}=\left(0.49 \pm 0.30_{\text {stat }} \pm 0.14_{\text {syst }}\right) \%
$$

This result does not cofirm the evidence for direct $C P$ violation in the charm sector previously reported in other analyses. 

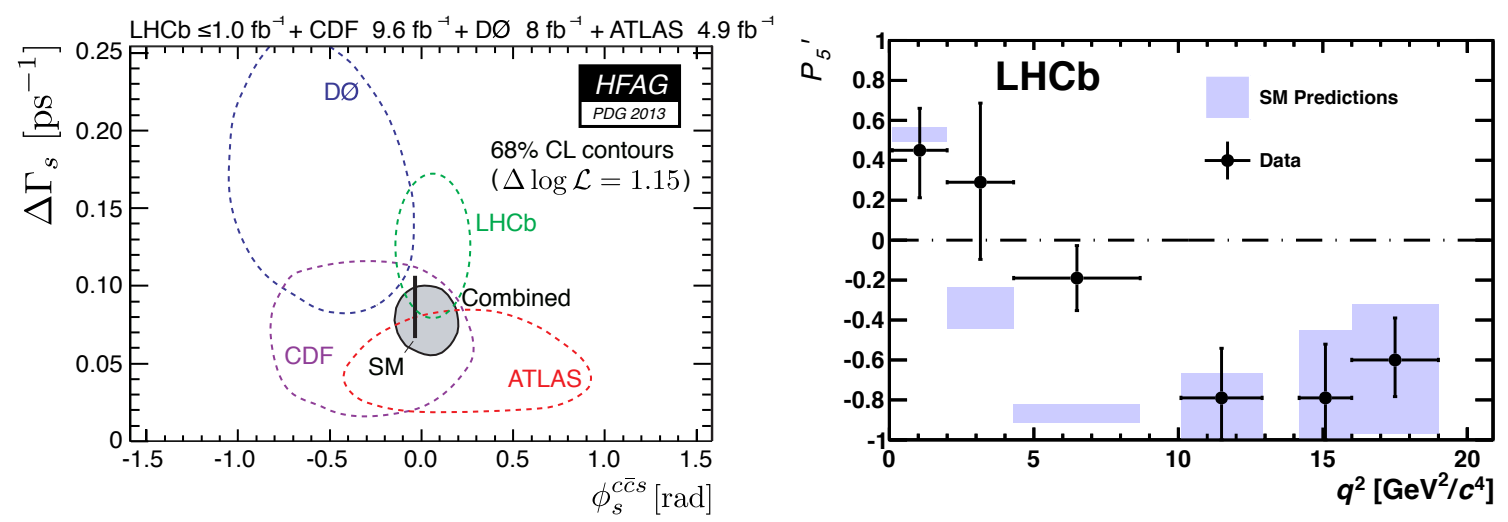

Figure 2: Left: $68 \%$ confidence level $(\mathrm{CL})$ contours in the $\left(\phi_{s}, \Delta \Gamma_{s}\right)$ plane, showing the measurements from single experiments and their combination; the thin rectangle represents the SM prediction [http://wWw.slac.stanford.edu/xorg/hfag/osc/PDG_2013/\#BETAS]. Right: Measured values (black points) of the form-factor independent observable $P_{5}^{\prime}$ in the decay $B^{0} \rightarrow K^{* 0} \mu^{+} \mu^{-}$, compared with SM predictions (blue bands) [10].

\subsection{First observation of direct $C P$ violation in $B_{s}$ mesons}

The direct $C P$-violating asymmetry in $B_{(s)}^{0} \rightarrow K \pi$ decays was measured by LHCb using data corresponding to an integrated luminosity of $1.0 \mathrm{fb}^{-1}$ collected in 2011 at a centre-of-mass energy of $7 \mathrm{TeV}$. After correcting the raw values for instrumental and production effects, the asymmetries were measured to be

$$
\begin{aligned}
& A_{C P}\left(B_{s}^{0} \rightarrow K^{-} \pi^{+}\right)=0.27 \pm 0.04_{\text {stat }} \pm 0.01_{\text {syst }} \\
& A_{C P}\left(B^{0} \rightarrow K^{+} \pi^{-}\right)=-0.080 \pm 0.007_{\text {stat }} \pm 0.003_{\text {syst }}
\end{aligned}
$$

leading to the first observation of direct $C P$ violation in $B_{s}$ mesons and the most precise measurement to date in $B_{0}$ [2]. These results allow for a stringent test of the relation between the two asymmetries in the Standard Model (SM) [3] and show no discrepancy from the prediction.

\subsection{Measurement of the $B_{S} C P$-violating phase}

LHCb performed the single most precise measurement to date of the $B_{S} C P$-violating phase $\phi_{s}$, the decay width difference between the light and the heavy mass eigenstates $\Delta \Gamma_{s}=\Gamma_{L}-\Gamma_{H}$ and their average $\Gamma_{s}$, using flavour tagged $B_{s}^{0} \rightarrow J / \psi K^{+} K^{-}$and $B_{s}^{0} \rightarrow J / \psi \pi^{+} \pi^{-}$decays selected from data collected in 2011 at a centre-of-mass energy of $7 \mathrm{TeV}$ corresponding to an integrated luminosity of $1.0 \mathrm{fb}^{-1}$. A combined unbinned maximum likelihood fit to both event categories yielded [4]

$$
\begin{aligned}
\phi_{s} & =0.01 \pm 0.07_{\text {stat }} \pm 0.01_{\text {syst }} \mathrm{rad} \\
\Gamma_{s} & =0.661 \pm 0.004_{\text {stat }} \pm 0.006_{\text {syst }} \mathrm{ps}^{-1} \\
\Delta \Gamma_{s} & =0.106 \pm 0.011_{\text {stat }} \pm 0.007_{\text {syst }} \mathrm{ps}^{-1}
\end{aligned}
$$

A similar measurement using flavour tagged $B_{s}^{0} \rightarrow J / \psi K^{+} K^{-}$events was performed by ATLAS based on $4.7 \mathrm{fb}^{-1}$ of data at a centre-of-mass energy of $7 \mathrm{TeV}$ [5]. The available measurements and 

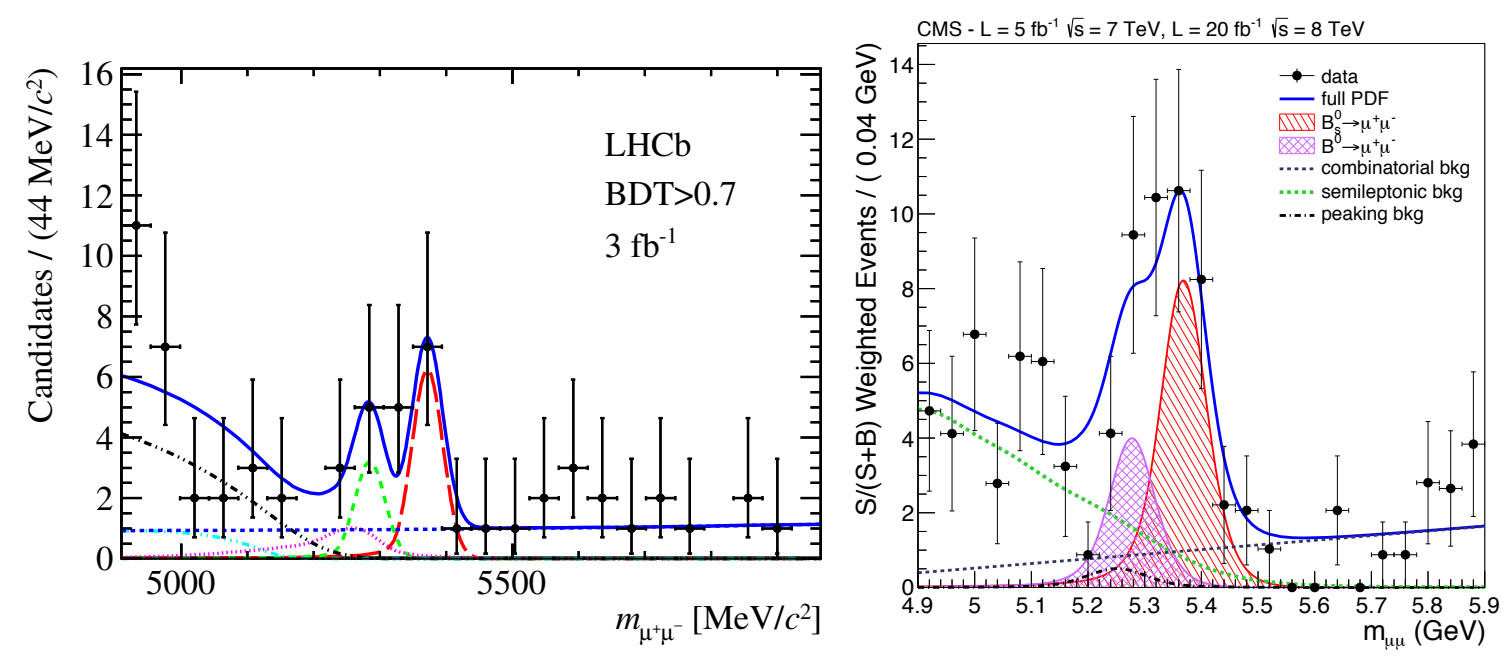

Figure 3: Di-muon invariant mass distribution of $B_{(s)}^{0} \rightarrow \mu^{+} \mu^{-}$candidates (black dots). The fit results and the different components are overlaid. Left: LHCb [12]. Right: CMS [13].

their combination are summarised in Figure 2 (left), all measurement are in agreement with the SM prediction.

\subsection{New observables in $b \rightarrow s \mu \mu$}

All experiments recently performed differential branching fraction measurements and angular analyses of the decay $B^{0} \rightarrow K^{* 0} \mu^{+} \mu^{-}$with $K^{*}(892)^{0} \rightarrow K^{+} \pi^{-}$, based on the full data sample collected in 2011 at a centre-of-mass energy of $7 \mathrm{TeV}$. This flavour-changing neutral current process is particularly interesting because in extensions of the SM new particles can modify the angular distributions of the decay products. All results agreed with the SM prediction within the uncertainties $[6,7,8]$.

In the newest $\mathrm{LHCb}$ analysis, based on the same data sample, the form-factor independent parameters defined in [9] were measured as a function of the invariant dimuon mass $q^{2}$, using folding techniques [10]. Agreement with the SM predictions was observed in 23 of the 24 measurements, while a local discrepancy of $3.7 \sigma$ was observed in the interval $4.30<q^{2}<8.68 \mathrm{GeV}^{2} / c^{4}$ for the observable $P_{5}^{\prime}$ (see Fig. 2 (right)). An interpretation of the experimental deviation in terms of physics beyond the Standard Model was provided in [11].

\subsection{Observation of $B_{s}^{0} \rightarrow \mu^{+} \mu^{-}$}

Searches for the rare decays $B_{s}^{0} \rightarrow \mu^{+} \mu^{-}$and $B^{0} \rightarrow \mu^{+} \mu^{-}$were performed by the LHCb and CMS experiments based on the complete datasets collected in 2011 and 2012 at centre-ofmass energies of 7 and $8 \mathrm{TeV}$ (corresponding to $3 \mathrm{fb}^{-1}$ for LHCb and $25 \mathrm{fb}^{-1}$ for CMS). Events were selected using boosted decision trees (BDT) and the number of candidate $B_{s}^{0} \rightarrow \mu^{+} \mu^{-}$and $B^{0} \rightarrow \mu^{+} \mu^{-}$events were extracted simultaneously via an unbinned maximum likelihood fit to the $m_{\mu \mu}$ distribution (see Fig. 3). While no significant evidence for $B^{0} \rightarrow \mu^{+} \mu^{-}$was found, both experiments observed an excess of $B_{s}^{0} \rightarrow \mu^{+} \mu^{-}$candidates with respect to the expectation from the background-only hypothesis with a significance of $4.0 \sigma$ and $4.3 \sigma$, respectively $[12,13]$. 

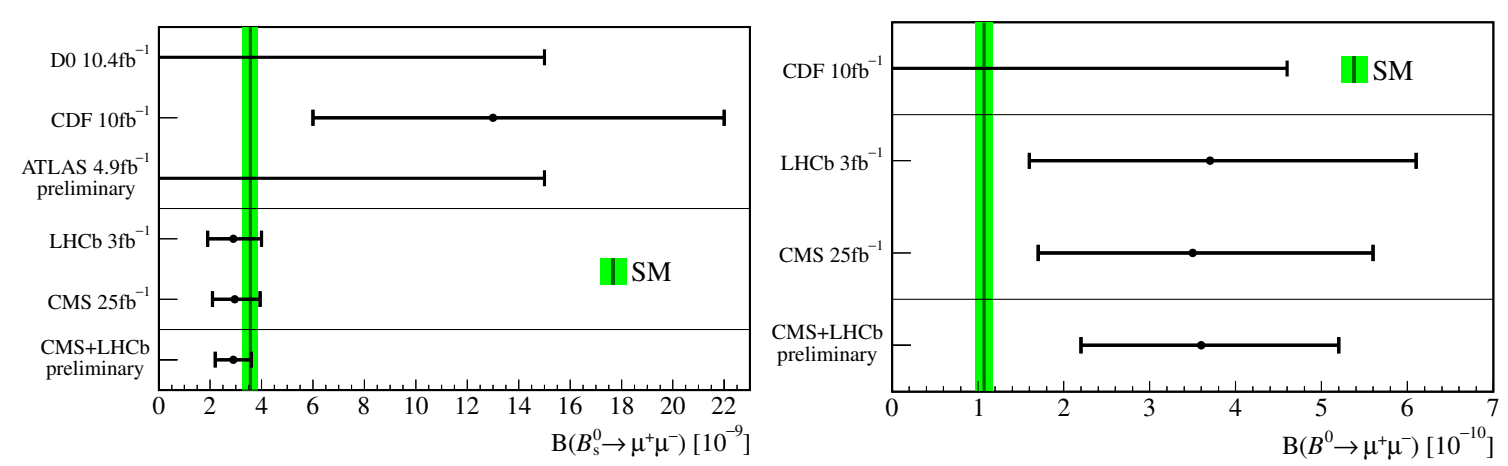

Figure 4: Comparison of previous results, the latest CMS and LHCb results, the combined value, and the SM prediction (vertical line) for the time-integrated branching fraction $B R\left(B_{s}^{0} \rightarrow \mu^{+} \mu^{-}\right)$(left) and $B R\left(B^{0} \rightarrow\right.$ $\mu^{+} \mu^{-}$) (right) [14].

A preliminary combination of the results was performed, yielding the first observation of $B_{s}^{0} \rightarrow \mu^{+} \mu^{-}$and the following values of the branching fractions (see Fig. 4):

$$
\begin{aligned}
& B R\left(B_{s}^{0} \rightarrow \mu^{+} \mu^{-}\right)=(2.9 \pm 0.7) \times 10^{-9}, \\
& B R\left(B^{0} \rightarrow \mu^{+} \mu^{-}\right)=\left(3.6_{-1.4}^{+1.6}\right) \times 10^{-10}
\end{aligned}
$$

\section{Top quark physics}

The LHC can be considered a top quark factory: millions of top quarks were produced in 2011 and 2012, allowing not only for precise measurements of the pair and single production cross sections, but also of many properties of this particle. In the following results concerning the $W t$ associated production and the top quark mass will be presented.

\subsection{First observation of $W t$ associated production}

While the single top production in the t-channel has long been established, the first evidence for $W t$ associated production was published by ATLAS in 2012 using data corresponding to an integrated luminosty of $2 \mathrm{fb}^{-1}$ at a centre-of-mass energy of $7 \mathrm{TeV}$ [15] and the s-channel production is still being searched for (see Fig. 5 (left)). CMS recently reported the first observation of $W t$ associated production in an analysis based on $12 \mathrm{fb}^{-1}$ of data collected in 2012 at a centre-of-mass energy of $8 \mathrm{TeV}$. Events with two leptons and a jet originating from a $b$-quark were fed to a boosted decision tree that was used to separate the signal from the background. The amount of signal was extracted from a binned maximum likelihood fit of the BDT distribution (see Fig. 5 (right)). The observed excess of $W t$ events was used to quantify the cross section and the CKM matrix element $\left|V_{t b}\right|$ to [16]

$$
\begin{aligned}
\sigma_{W t} & =23.4_{-5.4}^{+5.5} \mathrm{pb} \\
\left|V_{t b}\right| & =1.03 \pm 0.12_{\exp } \pm 0.04_{\text {th }} .
\end{aligned}
$$

\subsection{Measurement of the top quark mass}

Several measurements of the top quark mass in top-pair events were performed by ATLAS and CMS, the latest ones of which reached a precision close to the one of the Tevatron experi- 

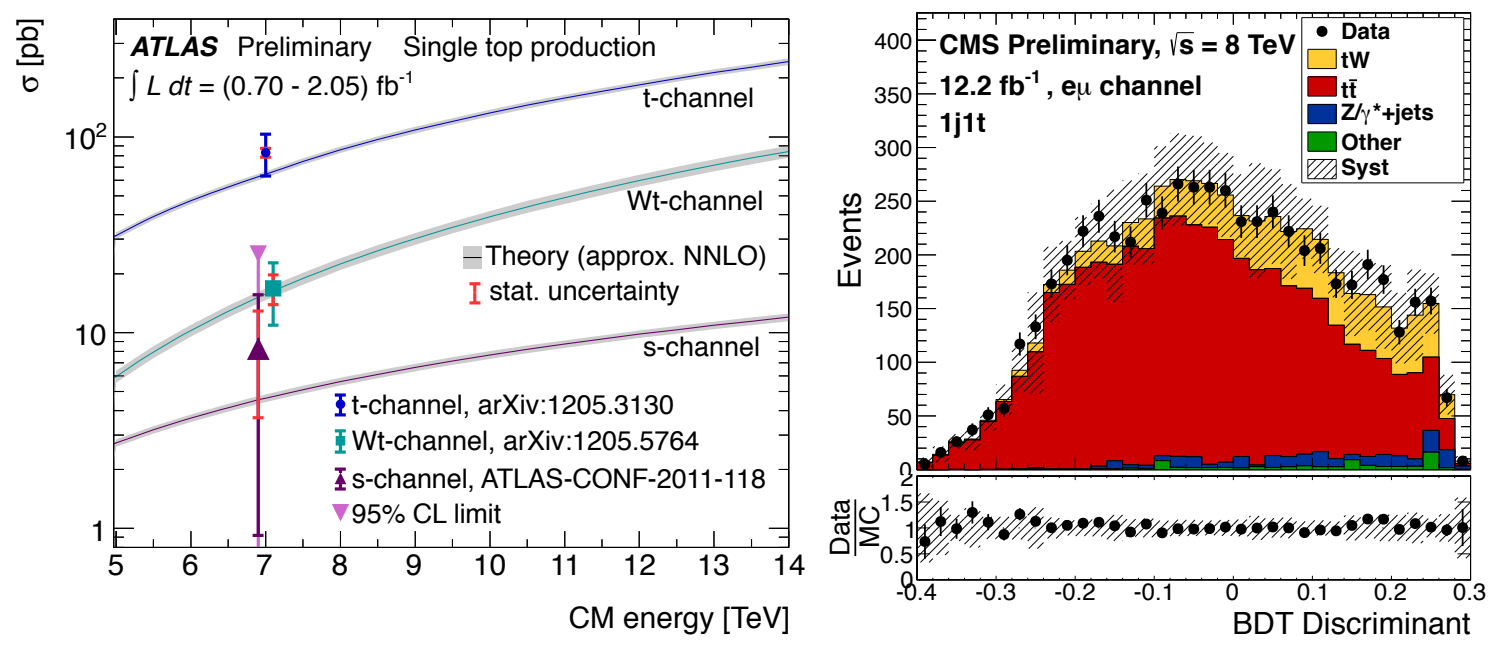

Figure 5: Left: Summary of ATLAS measurements of the single top production cross sections in various channels at a centre-of-mass energy of $7 \mathrm{TeV}$ compared to a theoretical calculation based on NLO QCD complemented with NNLL resummation. For the s-channel only an upper limit is shown [15]. Right: Distribution of the BDT discriminant for data and simulation in the signal region used for the CMS $W t$ cross section measurement [16].

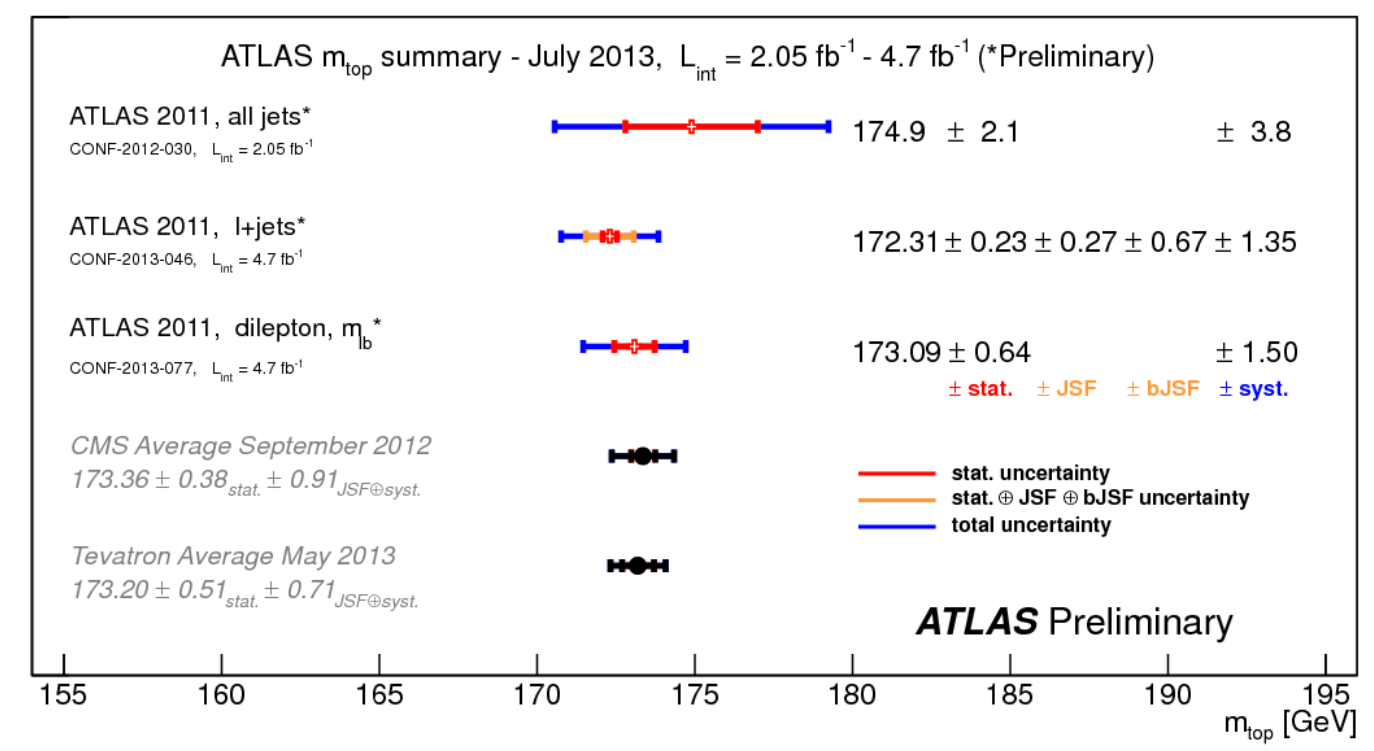

Figure 6: Summary of the latest ATLAS $m_{t}$ measurements, compared to the CMS and Tevatron combinations [18].

ments. The latest measurements by ATLAS made use of the template fit method and were based on the complete 2011 dataset. In the lepton+jets channel the top quark mass was extracted in a 3-dimensional fit simultaneously with global scale factors for the jet energy scale (JSF) and $b$-jet energy scale (bJSF), whose uncertainties are quoted separately in the result [17]. In the dilepton channel the $m_{\ell b}$ estimator was used, defined as the lowest average mass of the two lepton- $b$-jet pairs out of the two possible assignments of the $b$-jets to the selected leptons [18]. The latest results are summarised in Figure 6 and compared to the CMS and Tevatron combinations. A measurement 

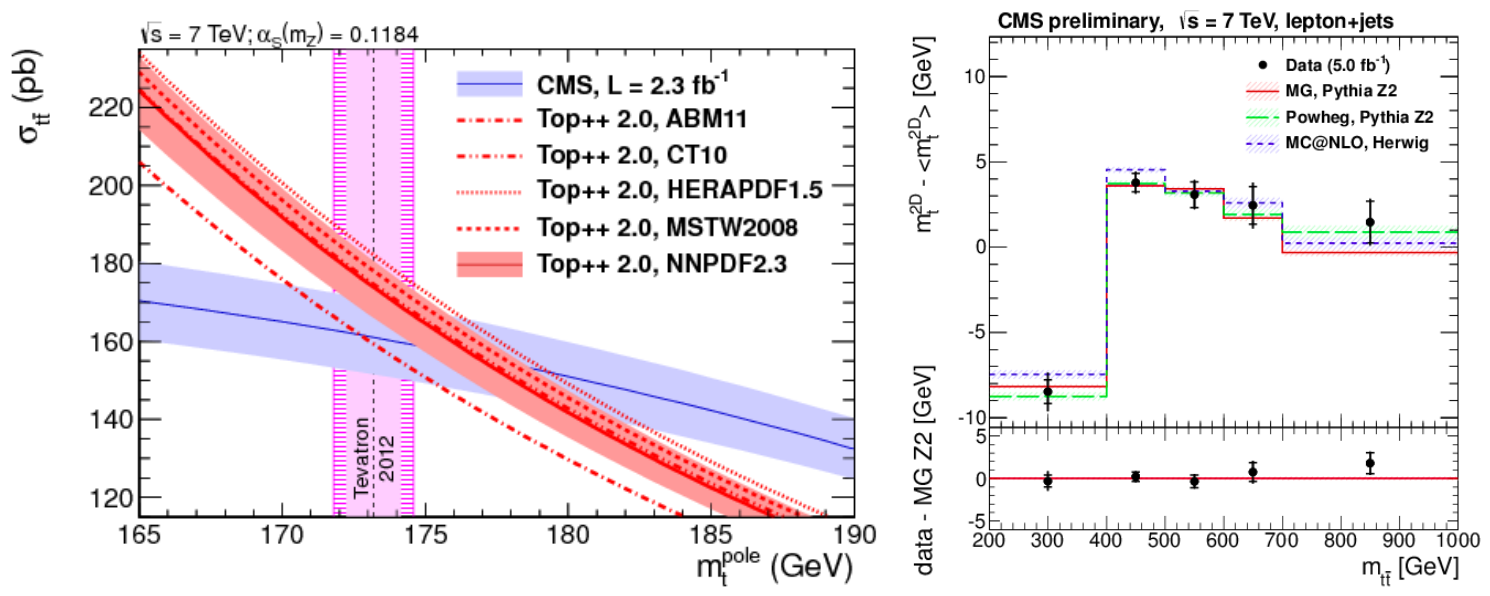

Figure 7: Left: Predicted $t \bar{t}$ cross section at NNLO+NNLL [20], as a function of the top-quark pole mass using five different NNLO PDF sets, compared to the cross section measured by CMS assuming $m_{t}=m_{t}^{\text {pole }}$ [21]. Right: Top quark mass measured by CMS in a 2D analysis (simultaneous determination of the jet energy scale) as a function of the invariant $t \bar{t}$ mass [23].

was recently performed by CMS in the all-jets channel using $3.5 \mathrm{fb}^{-1}$ of data at a centre-of-mass energy of $7 \mathrm{TeV}$. The mass was reconstructed for each event employing a kinematic fit of the jets to a $t \bar{t}$ hypothesis. A combination with previously published measurements in other decay modes by CMS yielded [19]

$$
m_{t}=173.54 \pm 0.33_{\text {stat }} \pm 0.96_{\text {syst }} \mathrm{GeV} .
$$

The top quark pole mass $m_{t}^{\text {pole }}$ can be extracted directly from the $t \bar{t}$ cross section to avoid the theoretical uncertainties on the top quark mass in the Monte Carlo (MC) simulation. The most precise measurement of this kind was performed by CMS using $2.3 \mathrm{fb}^{-1}$ of data at a centre-of-mass energy of $7 \mathrm{TeV}$ by comparing the measured cross section with the theoretical prediction [20] as a function of $m_{t}^{\text {pole }}$ for a fixed valuse of $\alpha_{s}=\alpha_{s}\left(M_{Z}\right)$, as shown in Figure 7 (left). The obtained measurement [21]

$$
m_{t}^{\mathrm{pole}}=176.7_{-3.4}^{+3.8} \mathrm{GeV}
$$

is compatible within the uncertainties with the MC-based ones and with a similar measurement by ATLAS [22].

Further preliminary results by CMS include a measurement of the dependence of the top quark mass on the event kinematics (see Fig. 7 (right)) [23], the extraction of the top quark mass from the lifetime of the $B$-hadrons produced in the decay of the top quark [24]

$$
m_{t}=173.5 \pm 1.5_{\text {stat }} \pm 1.3_{\text {syst }} \pm 2.6_{p_{T}(t)} \mathrm{GeV}
$$

and the measurement of the difference between the masses of the top and of the antitop quark [25]

$$
\Delta m_{t}=-272 \pm 196_{\text {stat }} \pm 122_{\text {syst }} \mathrm{MeV}
$$

which was found to be compatible with zero, as expected from $C P T$-invariance. 

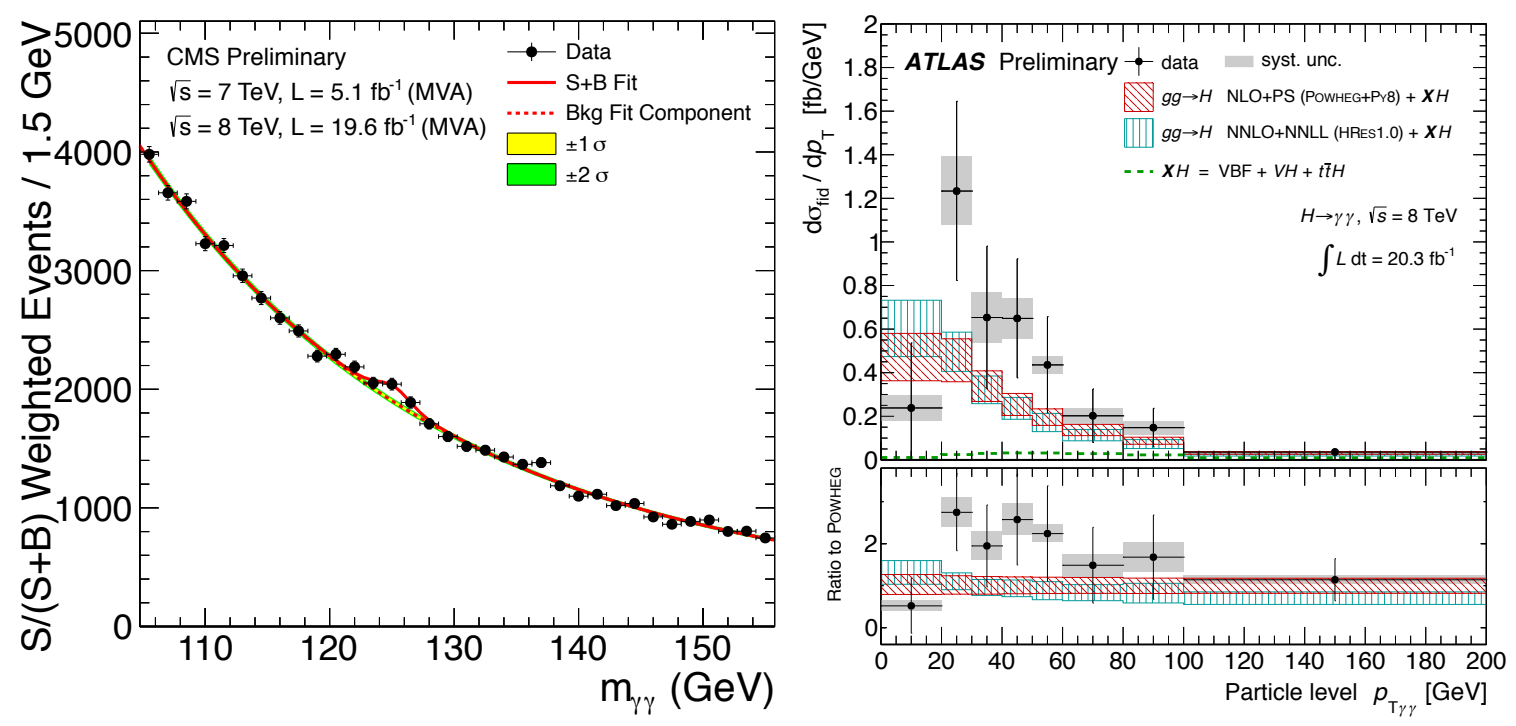

Figure 8: Left: Diphoton invariant mass distribution with each event weighted by the $S /(S+B)$ value of its category, for the multivariate CMS $H \rightarrow \gamma \gamma$ analysis [27]. Right: Differential $H \rightarrow \gamma \gamma$ cross section as a function of the diphoton $p_{T}$ measured by ATLAS [28].

\section{Higgs boson physics}

The discovery at the LHC of a particle compatible with the Higgs boson prediced by the Standard Model was announced in July 2012. Since then the precision of the signal strength $\left(\mu=\sigma / \sigma_{S M}\right)$ measurements was improved, more decay and production channels were investigated and properties of the new particle, such as spin and parity were tested for compatibility with the Standard Model prediction. An overview of the current status is given below.

\subsection{Measurement of $H \rightarrow \gamma \gamma$ signal strength and differential cross section}

ATLAS and CMS measured the signal strength for $H \rightarrow \gamma \gamma$ using the complete dataset collected in 2011 and 2012 by performing a fit in the distribution of the diphoton invariant mass $m_{\gamma \gamma}$ (see Fig. 8 (left)). The selected events were separated into several categories chosen to optimise the sensitivity. The measurements by ATLAS [26] and CMS [27] yielded

$$
\begin{aligned}
& \mu=1.55_{-0.28}^{+0.33} \text { and } \\
& \mu=0.78_{-0.26}^{+0.28},
\end{aligned}
$$

respectively. Furthermore ATLAS recently presented the first differential cross section measurement in the diphoton channel, based on $20 \mathrm{fb}^{-1}$ of data at a centre-of-mass energy of $8 \mathrm{TeV}$ (see Fig. 8 (right)). In general a good agreement between the unfolded data distributions and the MC predictions was observed [28].

\subsection{Measurement of $H \rightarrow Z Z$ and $H \rightarrow W W$ signal strength}

A mass fit in the invariant mass of the leptons $m_{4 \ell}$ was used by ATLAS and CMS to measure the signal strength in the $H \rightarrow Z Z^{*} \rightarrow 4 \ell$ channel (see Fig. 9 (left)). The events selected from 

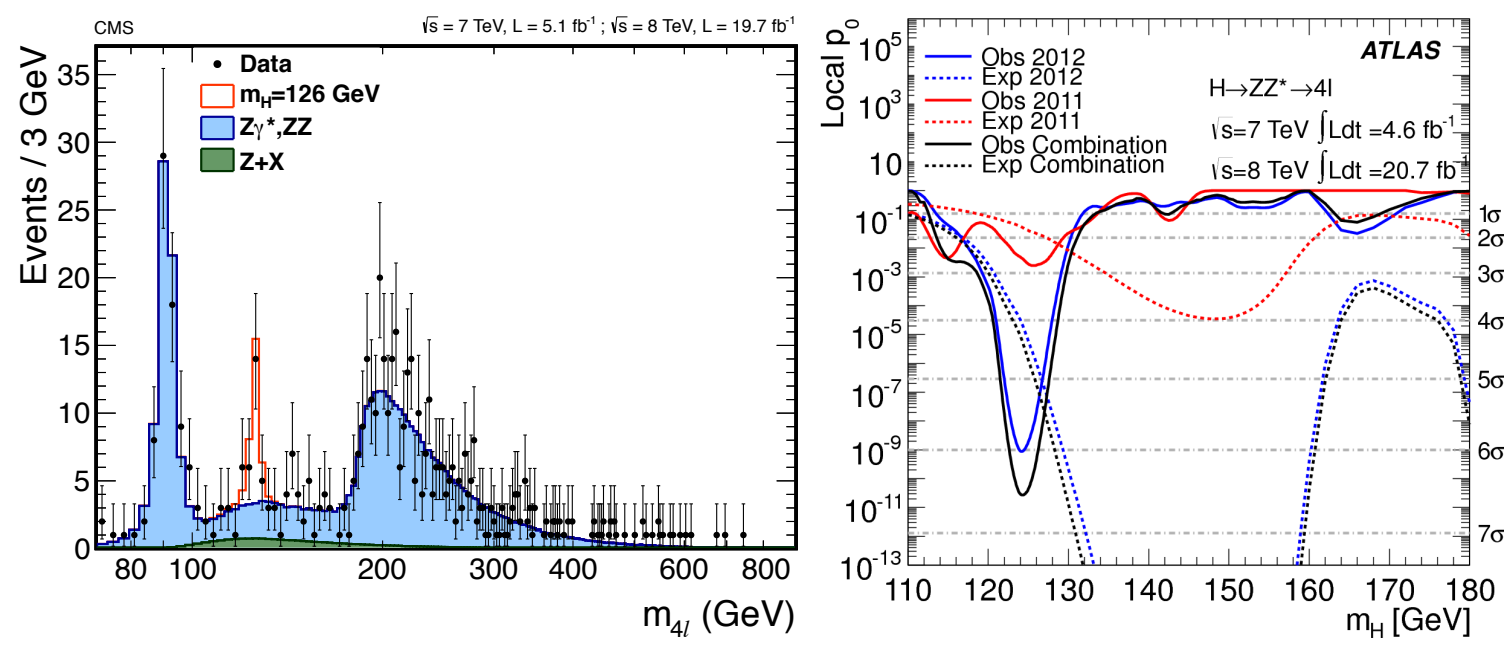

Figure 9: Left: Four-lepton invariant mass distribution for the sum of the $4 e, 4 \mu$, and $2 e 2 \mu$ channels [29]. Right: Observed local $p_{0}$ for the the $\sqrt{s}=7 \mathrm{TeV}$ data (red), the $\sqrt{s}=8 \mathrm{TeV}$ data (blue) and their combination (black), compared to the expectation for the SM Higgs boson as a function of the mass hypothesis [26].
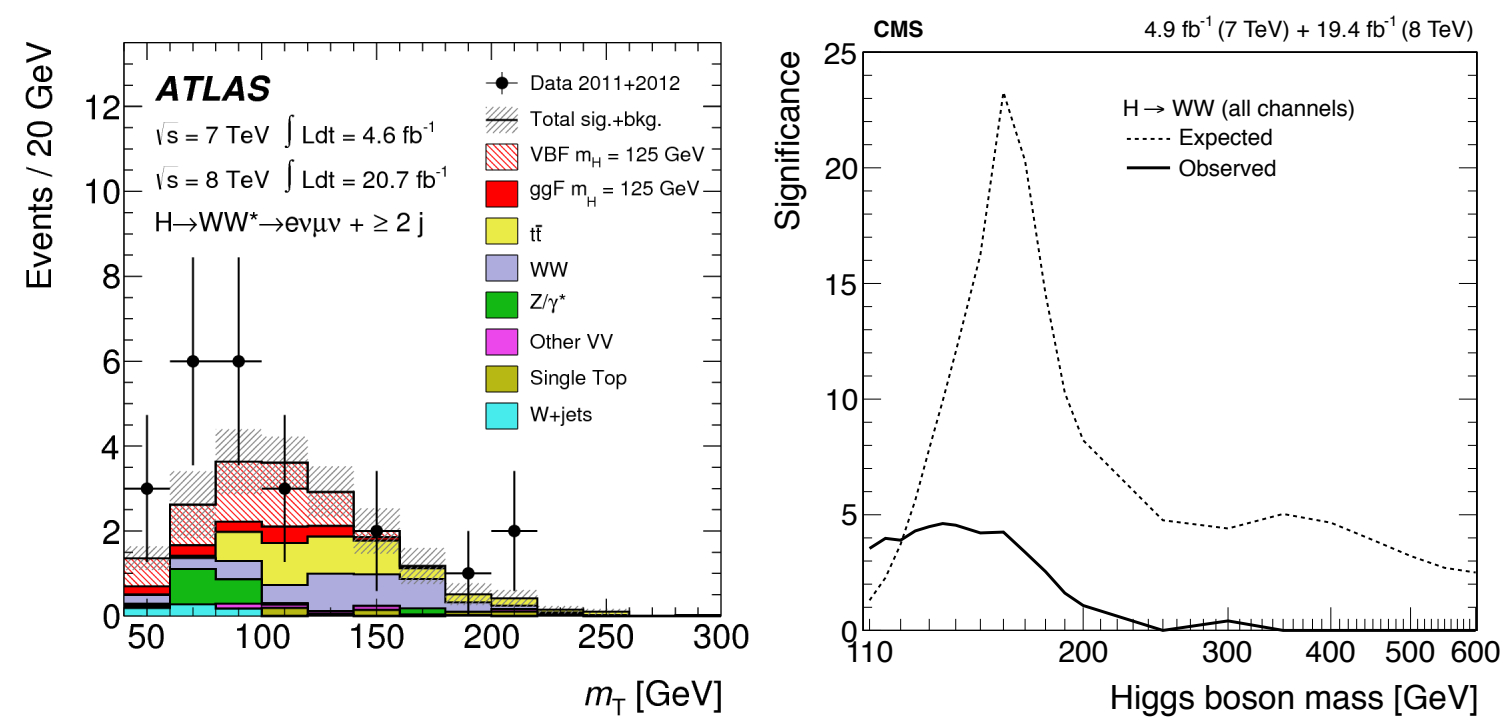

Figure 10: Left: Transverse mass distribution for events passing the full selection of the $H \rightarrow W W$ analysis for different-flavour final states with at least two additional jets [26]. Right: Observed and expected significances of the $H \rightarrow W W$ signal for each Higgs mass hypothesis [30].

the full 2011 and 2012 dataset were separated into categories dominated by different production mechanisms. The following values of $\mu$ were obtained by ATLAS [26] and CMS [29], respectively:

$$
\begin{aligned}
\mu & =1.43_{-0.35}^{+0.40}, \\
\mu & =0.93_{-0.25}^{+0.29} .
\end{aligned}
$$

The local probability $p_{0}$ to obtain the measured value of $\mu$ under the background-only hypothesis was quantified as a function of the Higgs mass $m_{H}$, as shown in Figure 9 (right). Each experiment excluded the background-only hypothesis by more than $6 \sigma$. 

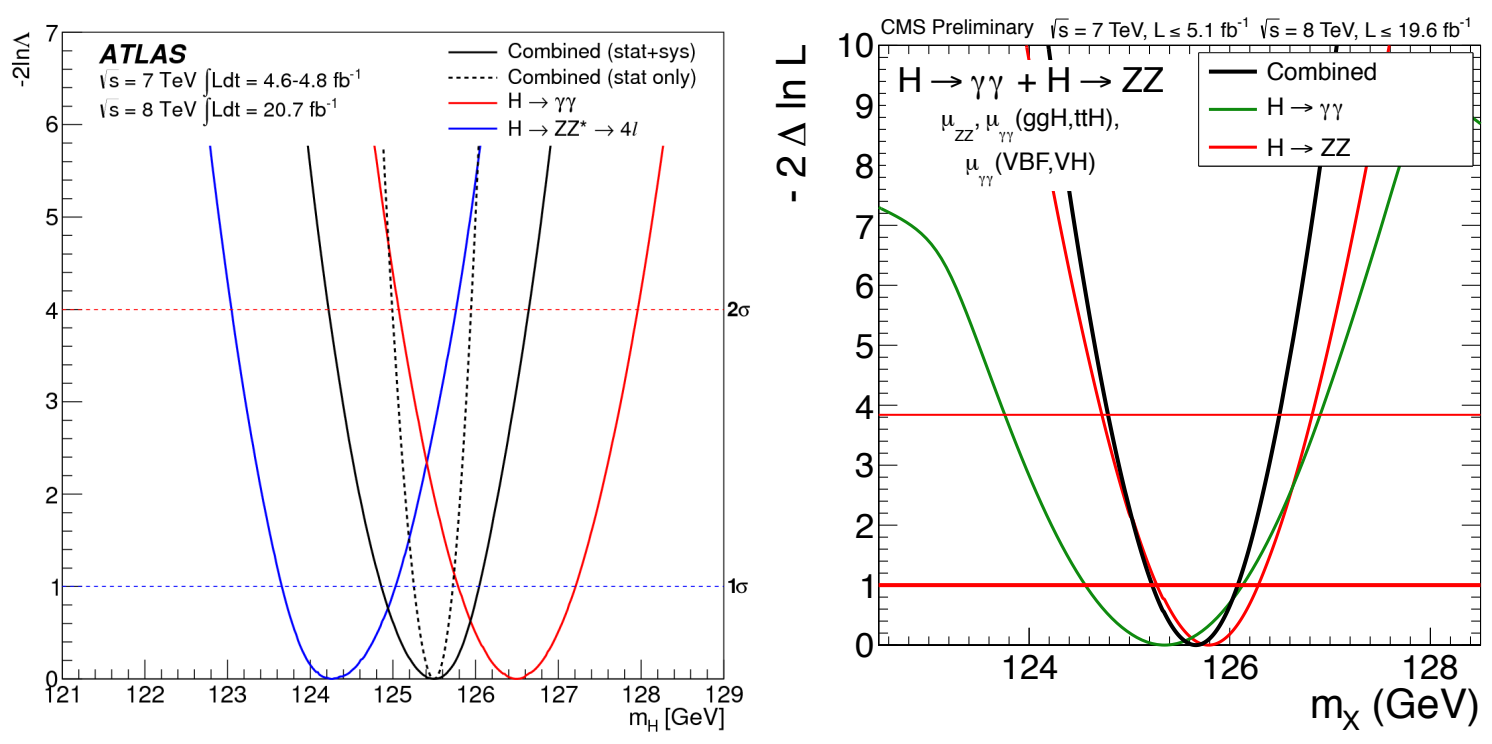

Figure 11: The profile likelihood ratio $-2 \ln \Lambda\left(m_{H}\right)$ as a function of $m_{H}$ for the $H \rightarrow \gamma \gamma$ and $H \rightarrow Z Z$ channels and their combination. Left: ATLAS [26]. Right: CMS [31].

Similarly, the $H \rightarrow W W$ signal strength was measured on the same dataset by dividing the events into categories dominated by different production (see e.g. the expected constribution for the vector boson fusion production mechanism (VBF) in Fig. 10 (left)) and decay modes and performing a fit in the transverse mass $m_{T}=\sqrt{\left(E_{T}^{\ell \ell}+E_{T}^{\text {miss }}\right)^{2}-\left|\vec{p}_{T}^{\ell \ell}+\vec{E}_{T}^{\text {miss }}\right|^{2}}$. The signal significance as a function of $m_{H}$ is shown in Figure 10 (right). For $m_{H}=125 \mathrm{GeV}$, the signal strength was measured to be

$$
\begin{aligned}
& \mu=0.99_{-0.28}^{+0.31} \\
& \mu=0.72_{-0.18}^{+0.20}
\end{aligned}
$$

for ATLAS [26] and CMS [30], respectively.

\subsection{Measurement of the Higgs boson mass}

The $H \rightarrow \gamma \gamma$ and $H \rightarrow Z Z$ analyses provided also measurements of the Higgs boson mass and a combination of the two values was provided by each experiment. The corresponding profile likelihood ratio distributions are shown in Figure 11. The minima of the combinations were found at

$$
\begin{aligned}
& m_{H}=125.5 \pm 0.2_{\text {stat }}^{+0.5}{ }_{-0.6 y s t} \mathrm{GeV} \text { and } \\
& m_{H}=125.7 \pm 0.3_{\text {stat }} \pm 0.3_{\text {syst }} \mathrm{GeV}
\end{aligned}
$$

for ATLAS [26] and CMS [31], respectively. The apparent discrepancy between the ATLAS measurements in the two channels was investigated and the values were found to be compatible within $8 \%$. 

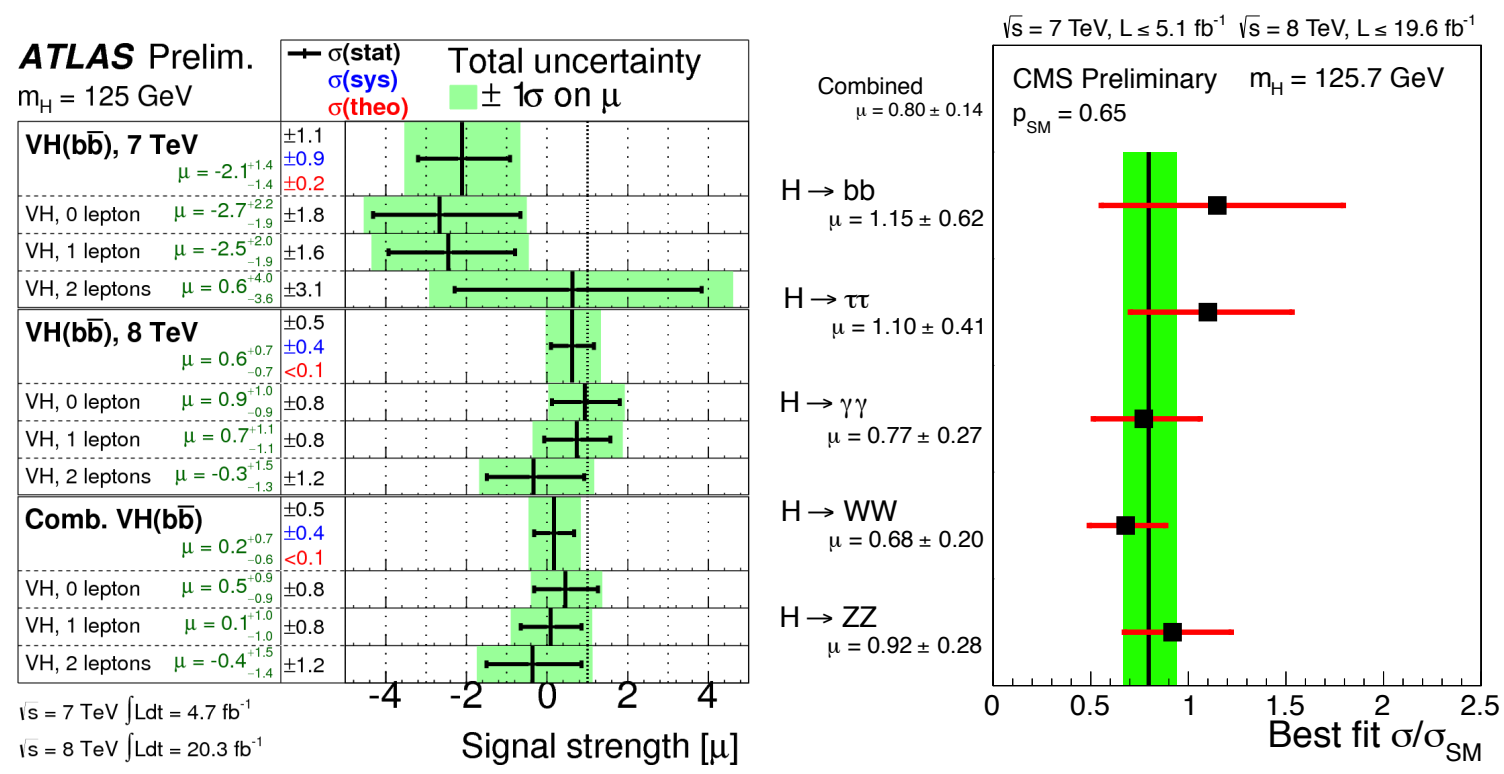

Figure 12: Left: Summary of the $V H$ with $H \rightarrow b \bar{b}$ signal strengths measured by ATLAS in different channels and datasets and their combination [32]. Right: Summary of the signal strengths (black points) measured by CMS for different decay modes of the Higgs boson and their combination (vertical line) [31].

\subsection{Measurement of the $H \rightarrow b \bar{b}$ signal strength}

The discovery of the Higgs boson was made in the decays into vector bosons. However, to confirm that the new particle is indeed the Higgs boson predicted by the SM, the coupling to the fermions need to be measured. Both ATLAS [32] and CMS [33] provided first results on the full 2011 and 2012 dataset for Higgs boson produced in association with a vector boson (VH) and decaying into a pair of $b$-quarks. ATLAS and CMS found the following values for the signal strength (see Fig. 12 (left)):

$$
\begin{aligned}
& \mu=0.2_{-0.6}^{+0.7} \text { and } \\
& \mu=1.0 \pm 0.5
\end{aligned}
$$

\subsection{Combination of the Higgs boson signal strength}

While ATLAS only combined the signal strengths measured in decays to vector bosons to [26]

$$
\mu=1.33_{-0.18}^{+0.21}
$$

CMS also included the fermionic decays (see Fig. 12), obtaining [31]

$$
\mu=0.80 \pm 0.14
$$

\subsection{Measurement of the Higgs boson couplings and properties}

The different production channels provide a first insight into the coupling of the new particle to vector bosons (via the VBF and VH channels) and to the top quark (via the loop in the gluongluon-fusion channel, ggF). Both experiments performed a 2-dimensional fit to both signal strength 

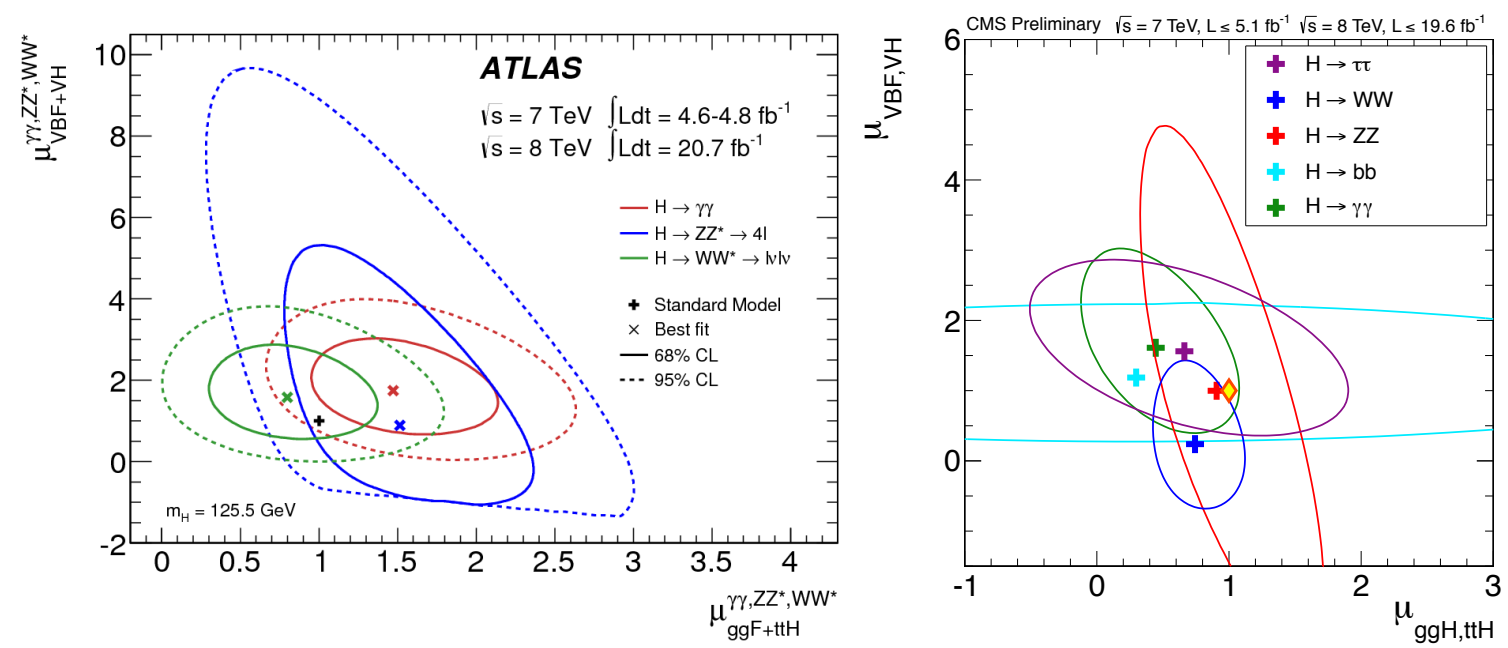

Figure 13: Likelihood contours for the Higgs boson signal strength in different production channels. Left: ATLAS [26]. Right: CMS [31].
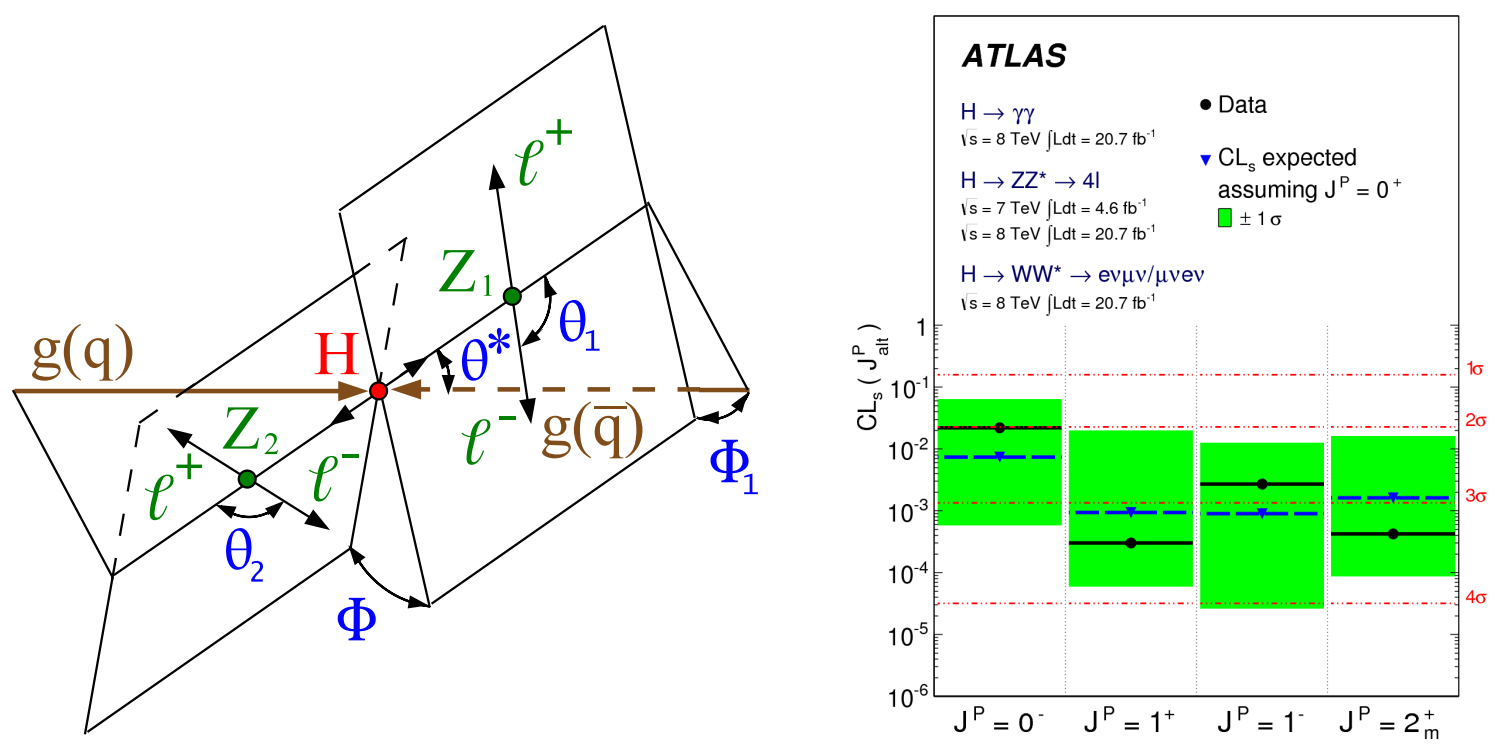

Figure 14: Left: Illustration of the production and decay of a Higgs boson [29]. Right: Expected (blue triangles/dashed lines/uncertainty as green band) and observed (black circles/solid lines) confidence level by ATLAS for alternative spin-parity hypotheses assuming a signal from a Higgs boson with $J^{P}=0^{+}$[34].

categories and compared the individual results in the different decay channels with the expectation from the SM. The results are shown in Figure 13 and they show, within the still large uncertainties, a good compatibility with the SM.

One of the unique features of the Higgs boson as predicted by the SM is its scalar nature, that can be tested in distributions of the production and decay angles as well as of the invariant masses (see Fig. 14 (left)). Several alternative hypotheses have been tested: a pseudoscalar, a vector, an axial vector, and a graviton-like tensor with minimal couplings. All hypotheses could be rejected in favour of the SM scalar as shown in Figure 14 (right). 

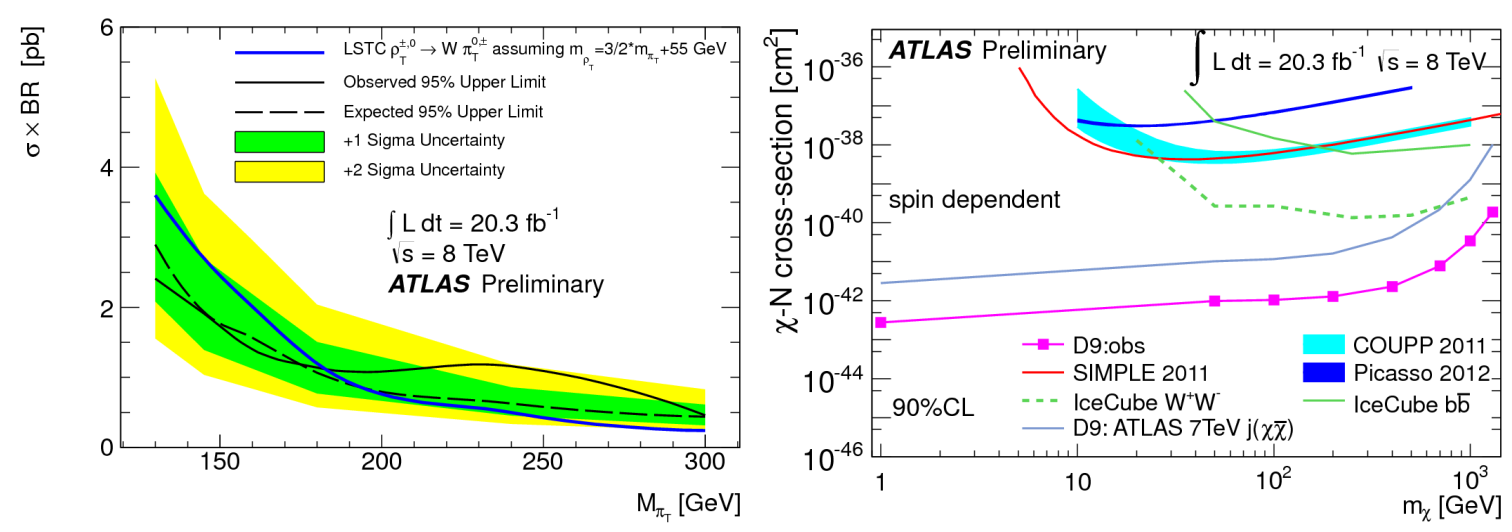

Figure 15: Left: Observed and expected 95\% CL upper limits on the $W \pi_{T}$ cross section as a function of the mass of the technipion $\pi_{T}$ [37]. Right: Observed $90 \% \mathrm{CL}$ limits on $\chi$-nucleon cross sections, compared to previous limits set by direct dark matter detection experiments and by the $7 \mathrm{TeV}$ monojet analysis [39].

\section{Searches for physics beyond the Standard Model}

The unprecedented centre-of-mass energy of the LHC was exploited to perform many searches for new heavy particles predicted by extensions of the Standard Model. Supersymmetry (SUSY) is the most prominent class of these models and specific searches were performed in different regions of the parameter space. The results of more general signature based searches were instead interpreted in terms of many different exotic models. Unfortunately so far none of the searches lead to an evidence for new particles and many exclusion limits were set on a vast range benchmark models. Only very few examples of recent results from signature based and from SUSY searches are presented below.

\subsection{Signature based searches}

Signature based searches are ideally model-independent and their results are presented in a way that allows for later re-interpretation in terms of new models. Since none of the searches lead to a significant excess of events above the background-only hypothesis, benchmark models were chosen in order to express the exclusion limits also in terms of e.g. the mass of a new particle.

A search for a dijet resonance produced in association with a leptonically decaying $W$ or $Z$ boson was performed by ATLAS on 2012 data corresponding to an integrated luminosity of $20 \mathrm{fb}^{-1}$. The observed distribution of the invariant dijet mass $m_{j j}$ was found to be in good agreement with the background expectation and upper limits on the $\rho_{T} \rightarrow V \pi_{T}$ cross section (with $V=W, Z$ ) in the low scale technicolor (LSTC) model [36] were set as a function of the technipion mass $m_{\pi_{T}}$ (see Fig. 15 (left)), which translate into a lower mass limit of $m_{\pi_{T}}>180 \mathrm{GeV}$ at 95\% CL [37].

In the hypothetical production via heavy particles, the much lighter intermediate decay products have a strong Lorentz boost and the final ones are collimated in a small cone. Therefore many searches use special tagging techniques to reconstruct hadronic decays of heavy bosons or of top quarks. Two recent analyses making use of these techniques were performed by CMS and ATLAS searching for $W$ or $Z$ tagged dijet resonances and for dark matter pair production with single $W$ or $Z$ tagged jets, respectively. Both searches used data at a centre-of-mass energy of $8 \mathrm{TeV}$ corresponding to an integrated luminosity of $20 \mathrm{fb}^{-1}$ and in neither of them a significant excess was observed 

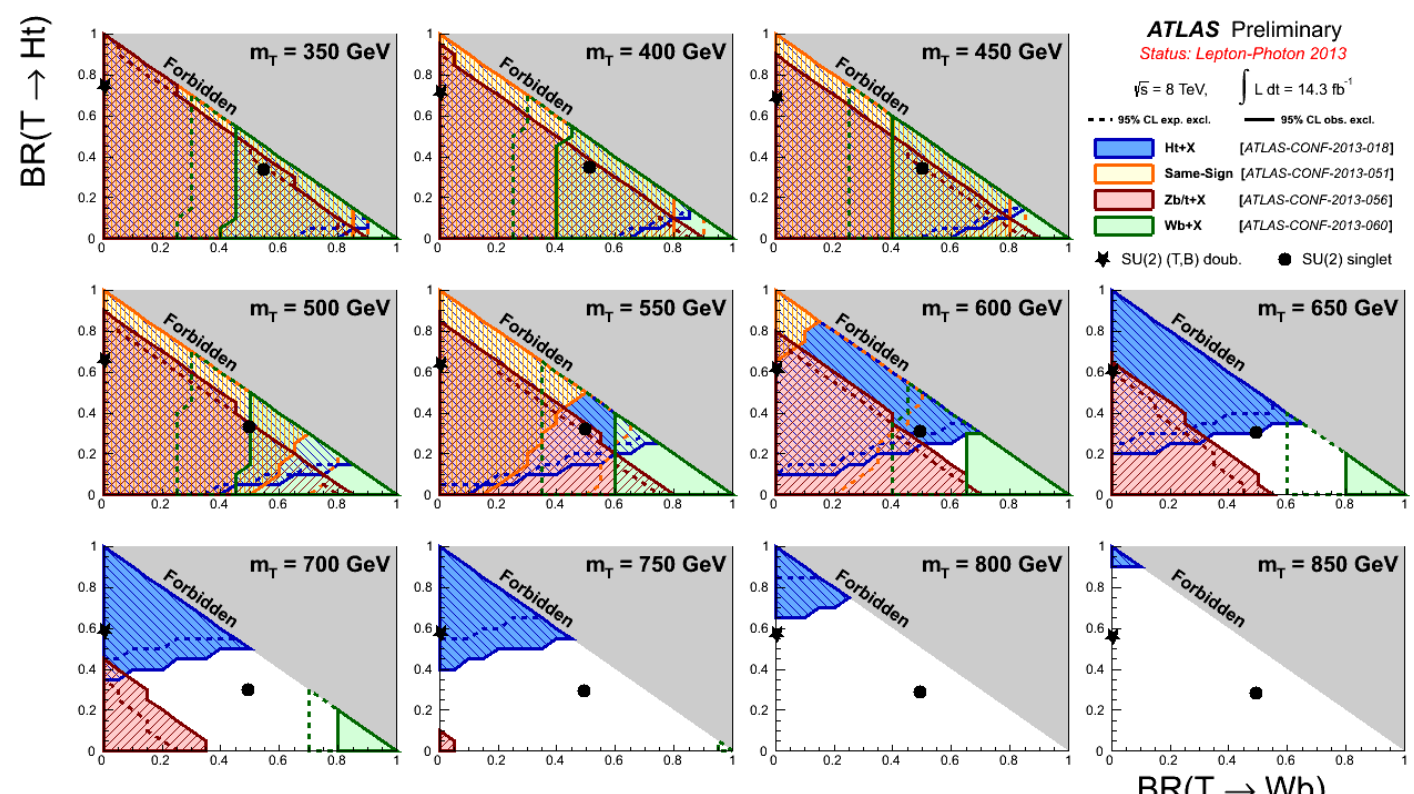

Figure 16: Summary of ATLAS searches for vector-like $T$ quarks: the regions excluded with $95 \%$ CL in different analyses are overlaid.

with respect to the background-only expectation. CMS set upper limits on the cross section for resonances decaying to $q W, q Z, W W, W Z$, or $Z Z$ final states, which were interpreted e.g. into the exclusion of the $W^{\prime}$, a heavy partner of the $W$ boson up to a mass of $1.73 \mathrm{TeV}$ at $95 \%$ CL [38]. ATLAS set limits on dark matter $(\chi)$ production in events with hadronically decaying $W$ or $Z$ boson and large missing transverse momentum. In the case of constructive interference between up-type and down-type contributions, the results set the strongest limits on the $\chi$-nucleon cross section (see Fig. 15 (right)) [39].

Searches for the pair production of heavy up-type $(T)$ or down-type $(B)$ quarks decaying preferentially to third generation SM particles were performed using data collected in 2012 at a centreof-mass energy of $8 \mathrm{TeV}$ corresponding to an integrated luminosity of $20 \mathrm{fb}^{-1}$ for CMS ( $T$-quark only) and of $14 \mathrm{fb}^{-1}$ for ATLAS. The decays $T \rightarrow t H, t Z, b W$ and $B \rightarrow b H, b Z, t W$ were considered and no assumptions were made on the branching fractions. All kinematic distributions were found to be compatible with the background-only hypothesis and upper limit were set on the production cross section as a function of the mass of the heavy quarks and of the decay branching fractions (see Fig. 16) [40, 41, 42, 43, 44]. The discovery of a SM-like Higgs boson strongly disfavours the existence of a fourth generation of chiral fermions, but weak-isospin singlets, doublets or triplets of vector-like quarks (VLQ) for which both chiralities have the same electroweak couplings, that appear in many extensions of the SM such as little Higgs and extra dimensional models, are compatible with the Higgs measurements. The cross section limits were interpreted as mass limits for specific models (fixed branching fraction values), but masses of the $T$ quark below $687 \mathrm{GeV}$ and of the $B$ quark below $350 \mathrm{GeV}$ could be excluded independently of the branching fraction values.

\subsection{SUSY serches}

After probing and excluding large parameter regions of the constrained Minimal Supersym- 

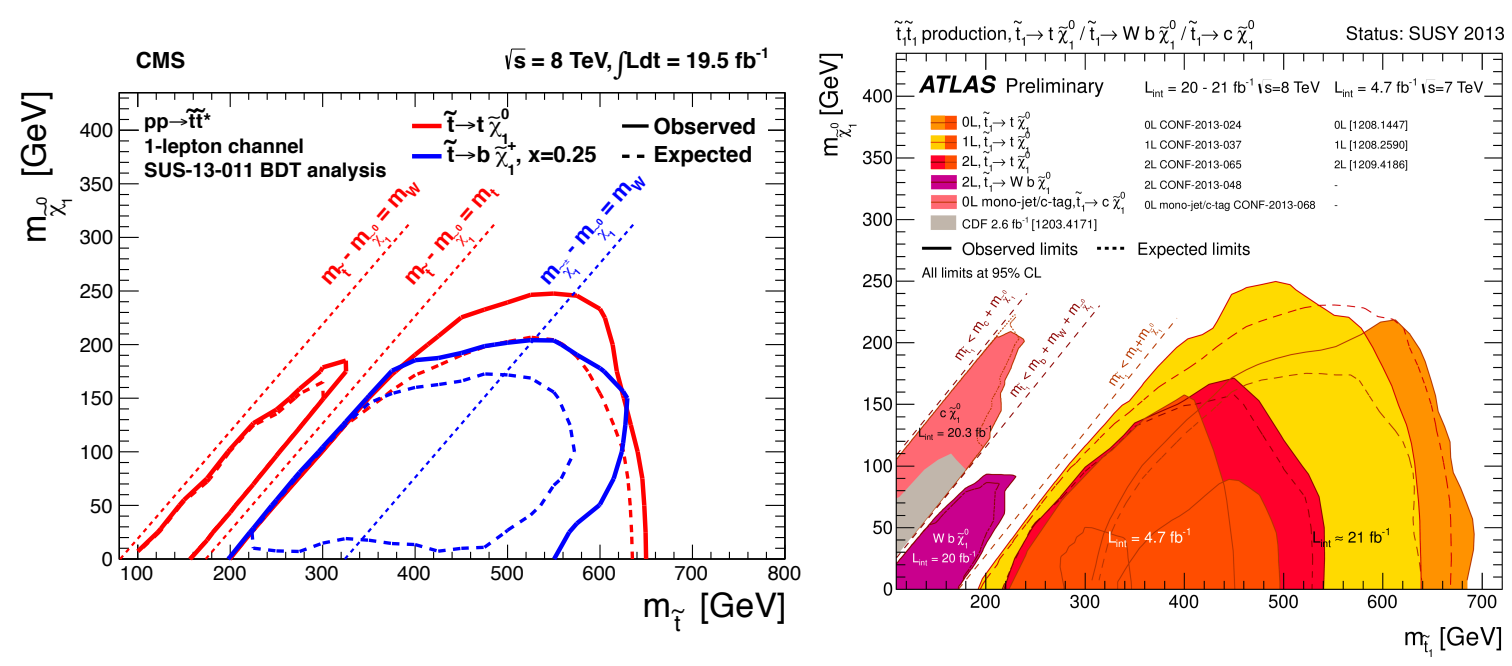

Figure 17: Exclusion limits at 95\% CL in the $\left(m_{\tilde{t}_{1}}, m_{\tilde{\chi}_{1}^{0}}\right)$ plane from dedicated searches for top squarks pair production. Left: CMS [37]. Right: ATLAS [39].

metric Standard Model (CMSSM), the searches performed by ATLAS and CMS are now moving towards simplified and natural SUSY models. Particularly interesting is the extension of the searches for the pair production of scalar top partners $\tilde{t}_{1}$ towards masses $m_{\tilde{t}_{1}}$ below the threshold for the decay into real top quarks. The decay $\tilde{t}_{1} \rightarrow W b \tilde{\chi}_{1}^{0}$ is the dominant process in the region $m_{b}+m_{W}<m_{\tilde{t}_{1}}-m_{\tilde{\chi}_{1}^{0}}<m_{t}$, while for even lower values of $m_{\tilde{t}_{1}}-m_{\tilde{\chi}_{1}^{0}}$ the dominant decay channel is $c \tilde{\chi}_{1}^{0}$. Using $20 \mathrm{fb}^{-1}$ of data collected in 2012 at a centre-of-mass energy of $8 \mathrm{TeV}$, CMS searched for pairs of $\tilde{t}_{1}$ decaying to $W b \tilde{\chi}_{1}^{0}$ in single lepton events with jets and large missing transverse energy [45]. On the corresponding dataset ATLAS performed a search for pairs of $\tilde{t}_{1}$ decaying to $W b \tilde{\chi}_{1}^{0}$ in events with two leptons [46] and two analyses to search for the decay into $c \tilde{\chi}_{1}^{0}$ using either a monojet topology or charm-tagging techniques [47]. All analyses found a good agreement between data and background-only expectations and set limits in the $\left(m_{\tilde{t}_{1}}, m_{\tilde{\chi}_{1}^{0}}\right)$ plane as shown in Figure 17.

\section{Conclusion}

The LHC and its experiments achieved great performance in the first 3 years of running, which allowed for a very high number of publications. In flavour physics an unprecedented precision was reached, previous tensions could be reconciled with the SM predictions, but a new exciting one appeared. The properties of top quarks could be investigated for the first time with very large statistics and a better understanding of the simulation is now the next step needed for further improvement. After the spectacular discovery last year, Higgs physics is already entering the phase of precision measurements, including e.g. differential cross section measurements and angular analyses. The many searches for physics beyond the Standard Model could not find any new particles and very stringent limits were set on a large variety of benchmark models.

At the moment the accelerator, the detectors and the analysis software are being upgraded to cope with the new running conditions starting in 2015. The sensitivity to heavy exotic particles will be extended towards higher masses by the centre-of-mass energy of $14 \mathrm{TeV}$, while the higher peak 
luminosity will make the experimental conditions harsher due to the higher pile-up. Many precision measurements are still in preparation on the existing data and new analyses will be prepared to be ready for discovery of the new physics "around the corner" right after the restart.

\section{References}

[1] LHCb Collaboration, Search for direct $C P$ violation in $D^{0} \rightarrow h^{-} h^{+}$modes using semileptonic $B$ decays, Phys. Lett. B 723 (2013) 33, [arXiv:1303.2614 [hep-ex] ] .

[2] LHCb Collaboration, First observation of $C P$ violation in the decays of $B_{s}^{0}$ mesons, Phys. Rev. Lett. 110 (2013) 221601, [arXiv:1304.6173 [hep-ex] ] .

[3] H. J. Lipkin, Is observed direct CP violation in $B_{(d)} \rightarrow K^{+} \pi^{-}$due to new physics? Check standard model prediction of equal violation in $B_{(s)} \rightarrow K^{-} \pi^{+}$, Phys. Lett. B 621 (2005) 126 , [hep-ph/0503022].

[4] LHCb Collaboration, Measurement of $C P$ violation and the $B_{s}^{0}$ meson decay width difference with $B_{s}^{0} \rightarrow J / \psi K^{+} K^{-}$and $B_{s}^{0} \rightarrow J / \psi \pi^{+} \pi^{-}$decays, Phys. Rev. D 87 (2013) 112010 , [arXiv:1304.2600 [hep-ex]].

[5] ATLAS Collaboration, Flavour tagged time dependent angular analysis of the $B_{s} \rightarrow J / \psi \phi$ decay and extraction of $\Delta \Gamma$ and the weak phase $\phi_{s}$ in ATLAS, ATLAS-CONF-2013-039, [http://cds.cern.ch/record/1541823].

[6] LHCb Collaboration, Differential branching fraction and angular analysis of the decay $B^{0} \rightarrow K^{* 0} \mu^{+} \mu^{-}$, JHEP 1308 (2013) 131, [arXiv:1304.6325 [hep-ex] ] .

[7] ATLAS Collaboration, Angular analysis of $B_{d}^{0} \rightarrow K^{* 0} \mu^{+} \mu^{-}$with the ATLAS Experiment, ATLAS-CONF-2013-038, [http://cds.cern.ch/record/1537961].

[8] CMS Collaboration, Angular analysis and branching fraction measurement of the decay $B^{0} \rightarrow K^{* 0} \mu^{+} \mu^{-}$, Phys. Lett. B 727 (2013) 77, [arXiv:1308.3409 [hep-ex] ] .

[9] S. Descotes-Genon, T. Hurth, J. Matias and J. Virto, Optimizing the basis of $B \rightarrow K^{*} \ell^{+} \ell^{-}$observables in the full kinematic range, JHEP 1305 (2013) 137, [ arXiv: 1303.5794 [hep-ph] ] .

[10] LHCb Collaboration, Measurement of form-factor independent observables in the decay $B^{0} \rightarrow K^{* 0} \mu^{+} \mu^{-}$, Phys. Rev. Lett. 111 (2013) 191801, [arXiv:1308.1707 [hep-ex] ] .

[11] S. Descotes-Genon, J. Matias and J. Virto, Understanding the $B \rightarrow K^{*} \mu^{+} \mu^{-}$Anomaly, Phys. Rev. D 88 (2013) 074002, [arXiv:1307.5683 [hep-ph] ] .

[12] LHCb Collaboration, Measurement of the $B_{s}^{0} \rightarrow \mu^{+} \mu^{-}$branching fraction and search for $B^{0} \rightarrow \mu^{+} \mu^{-}$decays at the LHCb experiment, Phys. Rev. Lett. 111 (2013) 101805, [arXiv:1307.5024 [hep-ex]].

[13] CMS Collaboration, Measurement of the $B_{(s)} \rightarrow \mu^{+} \mu^{-}$branching fraction and search for $B^{0} \rightarrow \mu^{+} \mu^{-}$with the CMS Experiment, Phys. Rev. Lett. 111 (2013) 101804 [arXiv:1307. 5025 [hep-ex] ].

[14] CMS and LHCb Collaborations, Combination of results on the rare decays $B_{(s)}^{0} \rightarrow \mu^{+} \mu^{?}$ from the CMS and LHCb experimentst, CMS-PAS-BPH-13-007, LHCb-CONF-2013-012, [http://cds.cern.ch/record/1564324].

[15] ATLAS Collaboration, Evidence for the associated production of $a \mathrm{~W}$ boson and a top quark in ATLAS at $\sqrt{s}=7 \mathrm{TeV}$, Phys. Lett. B 716 (2012) 142, [arXiv: 1205.5764 [hep-ex] ] . 
[16] CMS Collaboration, Single Top associated $\mathrm{W}$ production at $8 \mathrm{TeV}$ in the two lepton final state, CMS-PAS-TOP-12-040, [http://cds. cern. ch/record/1563135].

[17] ATLAS Collaboration, Measurement of the Top Quark Mass from $\sqrt{s}=7$ TeV ATLAS Data using a 3-dimensional Template Fit, ATLAS-CONF-2013-046, [http://cds.cern.ch/record/1547327].

[18] ATLAS Collaboration, Measurement of the Top Quark Mass in Dileptonic Top Quark Pair Decays with $\sqrt{s}=7$ TeV ATLAS Data, ATLAS-CONF-2013-077, [http://cds.cern.ch/record/1562935].

[19] CMS Collaboration, Measurement of the top-quark mass in all-jets $t \bar{t}$ events in pp collisions at $\sqrt{s}=7$ $T e V$, arXiv:1307.4617 [hep-ex].

[20] M. Czakon, P. Fiedler and A. Mitov, The total top quark pair production cross-section at hadron colliders through $O\left(\alpha_{S}^{4}\right)$, Phys. Rev. Lett. 110 (2013) 252004 [arXiv:1303.6254 [hep-ph] ].

[21] CMS Collaboration, Determination of the top-quark pole mass and strong coupling constant from the $t \bar{t}$ production cross section in pp collisions at $\sqrt{s}=7 \mathrm{TeV}$, arXiv:1307.1907 [hep-ex].

[22] ATLAS Collaboration, Determination of the Top-Quark Mass from the $t \bar{t}$ Cross Section Measurement in pp Collisions at $\sqrt{s}=7 \mathrm{TeV}$ with the ATLAS detector, ATLAS-CONF-2011-054, [http://cds.cern.ch/record/1342551].

[23] CMS Collaboration, Study of the dependence of the top-quark mass measurement on event kinematics, CMS-PAS-TOP-12-029, [http://cds. cern. ch/record/1521357].

[24] CMS Collaboration, Measurement of the top quark mass using the B-hadron lifetime technique, CMS-PAS-TOP-12-030, [http://cds.cern.ch/record/1563140].

[25] CMS Collaboration, Measurement of the top-antitop mass difference in pp collisions at $\sqrt{s}=8 \mathrm{TeV}$, CMS-PAS-TOP-12-031, [http://cds. cern.ch/record/1528156].

[26] ATLAS Collaboration, Measurements of Higgs boson production and couplings in diboson final states with the ATLAS detector at the LHC, Phys. Lett. B 726 (2013) 88, [arXiv: 1307.1427 [hep-ex] ].

[27] CMS Collaboration, Updated measurements of the Higgs boson at $125 \mathrm{GeV}$ in the two photon decay channel, CMS-PAS-HIG-13-001, [http://cds.cern.ch/record/1530524].

[28] ATLAS Collaboration, Differential cross sections of the Higgs boson measured in the diphoton decay channel with the ATLAS detector using 8 TeV proton-proton collision data, ATLAS-CONF-2013-072, [http://cds.cern.ch/record/1562925].

[29] CMS Collaboration, Measurement of the properties of a Higgs boson in the four-lepton final state, arXiv:1312.5353 [hep-ex].

[30] CMS Collaboration, Measurement of Higgs boson production and properties in the WW decay channel with leptonic final states, arXiv:1312.1129 [hep-ex].

[31] CMS Collaboration, Combination of standard model Higgs boson searches and measurements of the properties of the new boson with a mass near 125 GeV, CMS-PAS-HIG-13-005, [http://cds.cern.ch/record/1542387].

[32] ATLAS Collaboration, Search for the $b \bar{b}$ decay of the Standard Model Higgs boson in associated $(W / Z) H$ production with the ATLAS detector, ATLAS-CONF-2013-079, [http://cds.cern.ch/record/1562925]. 
[33] CMS Collaboration, Search for the standard model Higgs boson produced in association with a $W$ or a Z boson and decaying to bottom quarks, arXiv:1310.3687 [hep-ex].

[34] ATLAS Collaboration, Evidence for the spin-0 nature of the Higgs boson using ATLAS data, Phys. Lett. B 726 (2013) 120, [arXiv:1307.1432 [hep-ex] ] .

[35] CMS Collaboration, Properties of the observed Higgs-like resonance using the diphoton channel, CMS-PAS-HIG-13-016, [http://cds.cern.ch/record/1558930].

[36] E. Eichten, K. Lane, A. Martin and E. Pilon, Testing the Technicolor Interpretation of the CDF Dijet Excess at the 8-TeV LHC, Phys. Rev. D 86 (2012) 074015, [arXiv: 1206.0186 [hep-ph] ] .

[37] ATLAS Collaboration, Search for a dijet resonance produced in association with a leptonically decaying $W$ or $Z$ boson with the ATLAS detector at $\sqrt{s}=8 \mathrm{TeV}$, ATLAS-CONF-2013-074, [http://cds.cern.ch/record/1562930].

[38] CMS Collaboration, Search for heavy resonances in the W/Z-tagged dijet mass spectrum in pp collisions at $8 \mathrm{TeV}$, CMS-PAS-EXO-12-024, [http: / / cds. cern. ch/record/1563153] .

[39] ATLAS Collaboration, Search for dark matter in events with a hadronically decaying $W$ or $Z$ boson and missing transverse momentum in pp collisions at $\sqrt{s}=8 \mathrm{TeV}$ with the ATLAS detector, arXiv:1309.4017 [hep-ex].

[40] CMS Collaboration, Inclusive search for a vector-like T quark with charge 2/3 in pp collisions at $\sqrt{s}=8 \mathrm{TeV}$, arXiv:1311.7667 [hep-ex].

[41] ATLAS Collaboration, Search for heavy top-like quarks decaying to a Higgs boson and a top quark in the lepton plus jets final state in pp collisions at $\sqrt{s}=8 \mathrm{TeV}$ with the ATLAS detector, ATLAS-CONF-2013-018, [http: / / cds. cern. ch/record/1525525].

[42] ATLAS Collaboration, Search for anomalous production of events with same-sign dileptons and $b$ jets in $14.3 \mathrm{fb}^{1}$ of pp collisions at $\sqrt{\mathrm{s}}=8 \mathrm{TeV}$ with the ATLAS detector, ATLAS-CONF-2013-051, [http://cds.cern.ch/record/1547567].

[43] ATLAS Collaboration, Search for pair production of new heavy quarks that decay to a $Z$ boson and a third generation quark in pp collisions at $\sqrt{s}=8 \mathrm{TeV}$ with the ATLAS detector, ATLAS-CONF-2013-056, [http: / / cds. cern. ch/record/1557773].

[44] ATLAS Collaboration, Search for pair production of heavy top-like quarks decaying to a high- $p_{T} W$ boson and a $b$ quark in the lepton plus jets final state in pp collisions at $\sqrt{s}=8$ TeV with the ATLAS detector, ATLAS-CONF-2013-060, [http: / / cds. cern.ch/record/1557777].

[45] CMS Collaboration, Search for top-squark pair production in the single-lepton final state in pp collisions at $\sqrt{s}=8 \mathrm{TeV}$, Eur. Phys. J. C 73 (2013) 2677, [arXiv:1308.1586 [hep-ex] ] .

[46] ATLAS Collaboration, Search for direct top squark pair production in final states with two leptons in $\sqrt{s}=8 \mathrm{TeV}$ p collisions using $20 \mathrm{fb}^{1}$ of ATLAS data, ATLAS-CONF-2013-048, [http://cds.cern.ch/record/1547564].

[47] ATLAS Collaboration, Search for pair-produced top squarks decaying into charm quarks and the lightest neutralinos using $20.3 \mathrm{fb}^{1}$ of pp collisions at $\sqrt{\mathrm{s}}=8 \mathrm{TeV}$ with the ATLAS detector at the LHC, ATLAS-CONF-2013-068, [http: / / cds . cern. ch/record/1562880] . 